\title{
La migración femenina y los cambios en las relaciones de género en las familias: el caso de las transmigrantes colombianas en Italia*
}

\section{Alessandra Ciurlo**}

\section{RESUMEN}

En las migraciones internacionales, los movimientos poblaciones de mujeres son muy significativos, no solo por el número de personas que involucran, sino también porque presentan características propias que los distinguen de los movimientos poblacionales masculinos. La perspectiva de género aplicada a los fenómenos migratorios ha sido muy útil para demostrar que las migraciones no son neutrales en cuanto al sexo, y entender en profundidad las migraciones femeninas.
Este artículo se focaliza en las migraciones colombianas hacia Italia, un fenómeno todavía poco estudiado pero que presenta particularidades interesantes por su fuerte heterogeneidad, por la elevada presencia femenina y la variedad de itinerarios de las mujeres migrantes. A partir de la perspectiva de género, pretende observar las diferencias entre la experiencia de hombres y mujeres durante la migración. Ello para desentrañar algunas de las desigualdades de género y su intersección con otras desigualdades que derivan de algunas categorías sociales que intervienen durante la migración.

* Este artículo se basa en una investigación realizada en Italia en el marco de la tesis doctoral de la autora en 2012: "Migración colombiana hacia Italia: un estudio exploratorio sobre las familias transnacionales", Roma, Pontificia Università Gregoriana. Posteriormente, el texto de la tesis fue publicado en el libro Migración colombiana hacia Italia a la luz del género y de la familia transnacional (Ciurlo, 2013).

** PhD en Ciencias Sociales. Docente de investigación social en la Pontificia Università Gregoriana, Roma (Italia). aciurlo@yahoo.it

Recibido: 27 de marzo de 2015 / Modificado: 12 de junio de 2015 / Aceptado: 13 de junio de 2015.

Para citar este artículo

Ciurlo, A. (2015). La migración femenina y los cambios en las relaciones de género en las familias: el caso de las transmigrantes colombianas en Italia. OASIs, 21, 55-79. DOI: http://dx.doi.org/10.18601/16577558.n21.04 
Se focaliza en la familia, un observatorio importante donde las relaciones de género se manifiestan con gran determinación, pero donde se suceden cambios a partir de la migración, que contribuyen a modificar la condición familiar y social de la mujer. En dichos flujos, aunque los cambios en las relaciones de género no apuntan hacia una sola dirección, se evidencian diferentes trayectorias de las mujeres en las que a menudo ellas son protagonistas activas, independientes y autónomas, logrando articular ventajas y desventajas presentes durante el proceso migratorio.

Palabras clave: migración femenina, Colombia, Italia, género, familia transnacional.

\section{Female migration and changes in the family gender relations: The case of transmigrants co- lombian women}

\section{ABSTRACT}

Women population migrations are very significant in the broader international migration phenomenon, not only because of the number of people involved, but also because they have their own particular characteristics which distinguish them from male migrations. Gender perspective, being applied to migration studies, has been very useful in demonstrating that migration is a gendered phenomenon and it has added depth to the specific understanding of female migration.

This article focuses on Colombian flows to Italy, a scarcely studied phenomenon with interesting particularities, such as: its strong heterogeneity, the high female presence, and the variety of itineraries female have. Framed within the gender perspective, this paper aims to observe the differences between men's and women's migration experiences, in order to define some of the gender inequalities and their intersection with other inequalities that arise from certain social categories involved during the migration.

It focuses on the family, an important place of analysis since within it gender relations are expressed with great determination and at the same time it changes with migration, contributing to modify women's social and family status. Changes in gender relations do not point only in one direction in such flows, as women have different trajectories showing that they are often active, independent and autonomous actors, able to articulate advantages and disadvantages present during the migration process.

Key words: Female migration, Colombia, Italy, gender, transnational family.

\section{INTRODUCCIÓN}

La migración femenina es un fenómeno de larga data, que hoy día involucra a aproximadamente la mitad de quienes participan en los movimientos poblacionales internacionales. A pesar de haberse reconocido una presencia consistente de mujeres en algunos flujos y contextos específicos, en general, tal presencia ha permanecido en una situación de escasa visibilidad por muchos años, y se ha tendido a representar a las mujeres migrantes como dependientes pasivas de las figuras masculinas 
y carentes de autonomía. Los estudios sobre las migraciones, en muchas ocasiones, no han asumido la importancia de considerar las diferencias según el sexo, que determinan cambios sustanciales en la migración de hombres y mujeres, y de donde emerge que estas últimas tienen un rol activo en dicha experiencia. Parella (2005, pp. 241-242) evidencia que los movimientos migratorios femeninos tienen una identidad propia, con causas y consecuencias diferentes, al jugar la mujer un rol económico y social diferente al del hombre, tanto en la esfera productiva como en la reproductiva, y a la vez en la sociedad de origen y en la de destino.

En este sentido, la perspectiva de género ha permitido entender más profundamente los movimientos poblacionales de mujeres y sus particularidades, y también desentrañar desigualdades entre hombres y mujeres que intervienen en los procesos migratorios. Los estudios bajo este paradigma han hecho evidente, asimismo, que, junto con las disparidades que derivan del género, interactúan otras categorías sociales que determinan distintas divisiones también entre mujeres. Al observarlas en conjunto, es posible definir con mayor claridad y entender en profundidad el fenómeno de la migración femenina.

Este artículo pretende observar algunas desigualdades de género y su intersección con otras desigualdades que intervienen en el proceso migratorio para determinar las diversas trayectorias de las mujeres. Focaliza la atención en las familias, ya que, como sostiene Anthias (2000, p. 16), la familia es uno de los ámbitos en que las relaciones de género se manifiestan con mayor determinación. Ello en razón de ser una unidad de reproducción y de transmisión cultural donde sus miembros tienen posicio- nes, roles, funciones y un acceso al poder de modo diferenciado.

El objetivo que se persigue es identificar las disparidades de género y otras disparidades presentes antes de partir, así como también otras, efecto mismo de la migración, en especial dentro de la familia. Se pretende esbozar cómo dentro de los grupos familiares se negocian las relaciones de género durante la experiencia migratoria, teniendo en cuenta que la migración implica en la familia la puesta en discusión de los roles dominantes, contribuyendo a desafiarlos, lo que conduce a procesos de cambio, y, para las mujeres en algunos casos, a formas de emancipación que contribuyen a modificar su condición no solo familiar, sino también social.

Para efectos del propósito señalado, y desde la perspectiva de género con atención al enfoque interseccional, se ha tomado como caso de estudio la migración colombiana hacia Italia. En dicho flujo, las mujeres tienen un rol importante no solo por su mayo número respecto a los hombres, sino también por aspectos cualitativos que las muestran como protagonistas activas, independientes y autónomas, logrando articular desventajas y oportunidades presentes durante el proceso migratorio, construyendo nuevas representaciones de lo femenino, y produciendo recorridos originales.

El artículo se basa en una investigación de tipo cuantitativo, conducida en el año 2009, mediante un cuestionario estructurado a todo lo largo del territorio italiano, que adoptó la estrategia comparativa de los datos entre hombres y mujeres. La preparación de dicho instrumento requirió de una serie de entrevistas cualitativas preliminares a informantes clave, de las que 
emergieron indicaciones importantes. La encuesta fue aplicada a una muestra de 150 personas en diversos grupos de agregación informal, en los consulados de Roma y Milán, y mediante la ayuda de grupos informales presentes en las redes sociales. Se trata de una muestra de tipo no probabilístico por cuotas, en las que las variables estructurales son la edad y el sexo; con una proporción de mujeres del 64,7 \% sobre el total.

Además de los elementos metodológicos presentados, y para comprender a cabalidad el fenómeno en cuestión, antes de proceder con el marco teórico, cabe mencionar que la migración internacional colombiana tiene ya varias décadas de antigüedad. A pesar de que históricamente gran parte de los flujos de la misma se han dirigido a Estados Unidos, a finales de los años noventa, una oleada considerablemente grande y cada vez más heterogénea respecto al pasado, derivada de los diversos problemas socioeconómicos y políticos que ha vivido el país, ha diversificado los destinos habituales y se ha dirigido en buena parte a Europa. En especial hacia España y, en menor medida, a otros países, entre los cuales Italia. Allí el colectivo colombiano está constituido por $19.661^{1}$ personas y otra pequeńa porción se encuentra en condiciones irregulares de estadía.

\section{LA PERSPECTIVA DE GÉNERO Y LAS MUJERES MIGRANTES}

La feminización de las migraciones ha comportado en el ámbito teórico y metodológico de los estudios migratorios, la búsqueda de una perspectiva de análisis capaz de explicar las evidencias empíricas del comportamiento diferenciado de hombres y mujeres. Son diversos los estudios que han concluido que el fenómeno migratorio no es asexuado, y que, como en otros fenómenos sociales, el género se constituye en principio organizador: allí la mujer no tiene un rol marginal sino, muy por el contrario, plenamente protagónico (Hondagneu Sotelo, 1994; Morokvasic, 1984; Phizacklea, 1983).

A partir de la explicación de Scott (1986, pp. 288-290), el género es un elemento constitutivo de las relaciones sociales basadas en las diferencias que distinguen los sexos, siendo además una forma primaria de relaciones significantes de poder. Las diferencias biológicas entre los sexos, de este modo, constituyen desigualdades entre hombres y mujeres, y sitúan en el terreno de lo simbólico, cultural e histórico los determinantes de la desigualdad. El género tiene, por tanto, una valencia como construcción social de las desigualdades y, a la vez, como elemento estructurador de las relaciones sociales.

Carling (2005, p. 6) sostiene que las relaciones de género producen y reproducen las prácticas sociales definiendo los roles de hombres y mujeres. En el campo de las migraciones, estas tienen un gran impacto pues afectan la dimensión, dirección y composición de los flujos migratorios, así como también la experiencia individual de quien migra. Morokvasic (2008) identifica diferencias sustanciales entre

1 Datos del 1 de enero del 2014 (ISTAT - http://stra-dati.istat.it/). 
hombres y mujeres durante el proceso migratorio; ello es observable en lo que concierne a los motivos para emigrar y la relación con las familias y los hogares (Hondagneu y Ávila, 1997; Oso, 2000; Zlotnik, 1992, 2003; Zontini, 2005); las conexiones con las personas en el país de origen y la manera como se mantienen dichas relaciones, y las responsabilidades que asumen las y los migrantes (Pedone 2006); la importancia dada a la identidad nacional (Anthias, 2000); la experiencia de vida en el nuevo contexto; la inserción laboral (Hochschild, 2001; Parreńas, 2001); el protagonismo en el rol económico y productivo (Kofman et al., 2000) y la orientación hacia el retorno.

Si bien el género como categoría de análisis crítico de las desigualdades y de las prácticas sociales donde hombres y mujeres elaboran, construyen y recrean sus experiencias en la migración resulta extremadamente útil, es también cierto que durante ańos, como sugiere Morokvasic (1984), se tendió a considerar a la mujer migrante desde una visión estereotipada, vinculándola a un único origen cultural simplificado, en últimas ligado a una "tradición" que se contraponía al modelo de la mujer moderna occidental y urbana, al cual esta debía tender idealmente para emanciparse. A partir del Black Feminism, se pone en evidencia que las mujeres no corresponden a una categoría universal y unitaria, y que existen también, además de las diferencias de género y de las relaciones de poder entre hombres y mujeres, otras diferencias que remiten a la clase social y a la raza y etnia, las cuales determinan fuertes disparidades no solo entre los sexos, sino entre las mismas mujeres (Brah, 2004). De allí la toma de conciencia de la no homogeneidad de la mujer migrante y de la doble o triple discriminación a que está expuesta (Campani, 2000). Discriminaciones que, sin embargo, como sugieren Anthias y Yuval Davis (1983; Yuval Davis, 2006), no deben sumarse entre sí, por el hecho de tener bases ontológicas diferentes. Estas se deben articular para poder comprender los varios niveles de dominación que sufren las mujeres migrantes en los distintos contextos. Desde este planteamiento, se desarrolla el enfoque interseccional que introduce Crenshaw (1991) cuando sostiene que las categorías de género, raza, etnia y clase, entre otras, "interseccionan" la vida de las personas de forma diferente en cada situación personal y grupo social, evidenciando estructuras de poder existentes en el seno de la sociedad que modelan las acciones y la posición social de los individuos. Es así que las personas pueden experimentar opresiones y privilegios de manera simultánea según las especificidades de cada situación y experiencia.

$\mathrm{El}$ análisis interseccional privilegia, por tanto, la contextualización. Como sostiene Kócsé (2011, p. 134), se focaliza en observar cómo interactúan algunos tipos específicos de distribuciones desiguales de poder, fundados en categorías socioculturales construidas como las antes mencionadas, y también con otras, como la sexualidad, la edad o la generación, las discapacidades, la nacionalidad y el idioma materno, que producen diferentes tipos de desigualdades sociales. Estas categorías producen experiencias únicas cuando se cruzan, y posicionan a las personas en los contextos migratorios de formas particulares.

Según Anthias (2013, p. 3), este enfoque ofrece un correctivo a la construcción 
esencialista de la identidad que homogeneiza las categorías sociales. Mediante el análisis interseccional se busca, por tanto, revelar las diversas identidades de las personas y reconocer los diferentes tipos de discriminación y desventajas que se dan como consecuencia de la combinación de dichas identidades, así como también de ventajas y del acceso a los derechos $\mathrm{y}$ a las diversas oportunidades.

La aproximación interseccional al fenómeno de las migraciones femeninas muestra la complejidad de las desigualdades que se dan durante el proceso migratorio; pone en evidencia los diferentes roles, estatus y posiciones sociales que ocupan las mujeres, sus posibilidades de reacción y de cambio; ayuda a superar la visión de las inmigrantes como víctimas con identidades pasivas (Guzmán Ordaz, 2011, p. 875), y contempla, además, la migración más allá del foco único de la sociedad receptora: de hecho, Anthias (2000, p. 16), respecto a las relaciones de género, sostiene que estas reunifican en una misma experiencia el contexto de origen y el de llegada. Ello evidencia la existencia de relaciones transnacionales y pone en cuestión el enfoque transnacional que, como explican Basch, Glick Shiller y Szanton Blanc (1994, p. 7), es el proceso mediante el cual los transmigrantes, forjan y mantienen relaciones sociales, económicas y políticas que conectan las sociedades de proveniencia y de destino, para crear espacios sociales transnacionales que atraviesan las fronteras de los Estados-nación.

Bajo este marco conceptual, en la observación de la migración femenina se examinan los diferentes estatus y posiciones de las mujeres durante el proceso migratorio, sin poder prescindir de la interrelación de los contextos implicados en la migración ni de las relaciones transnacionales que se instauran. Las familias son un observatorio importante para tal fin, ya que allí se pueden examinar las relaciones entre los individuos miembros de la familia, las relaciones con las comunidades e instituciones, e igualmente las relaciones macroestructurales que intervienen a nivel global en las migraciones, en donde se estructura lo que Mahler y Pessar (2001) han definido como gendered geographies of power. Observar a la familia en el marco de la migración femenina no significa asumir este como un espacio solo femenino, sino como un espacio de negociación de roles y de relaciones entre hombres y mujeres, donde se produce la construcción social de las identidades de los y las migrantes, que a su vez determinan y tienen implicaciones en la misma experiencia migratoria.

\section{¿QUIÉNES SON LAS MUJERES QUE EMIGRAN Y POR QUÉ LO HACEN?}

Para comprender la migración femenina y sus implicaciones, y siguiendo el enfoque interseccional, se evidencia la importancia de conocer a fondo las diversas características de las mujeres migrantes. En el colectivo colombiano en Italia -a diferencia de lo que ocurre en otros colectivos nacionales altamente feminizados presentes en el territorio italiano, en donde las mujeres tienen características similares entre $\mathrm{si}^{2}-$, se hace

\footnotetext{
2 El peruano y el ecuatoriano entre los latinoamericanos, el ucranio entre los países de Europa del Este: en ellos es más fácil delinear los modelos migratorios que adoptan las mujeres.
} 
difícil definir una tipología específica de las colombianas que emigran, pues se trata de un grupo marcadamente heterogéneo en cuanto a la edad y a la proveniencia regional, si bien una buena parte $(72,1 \%)$ proviene de zonas desarrolladas del país como el área metropolitana de Bogotá, Antioquia, Valle del Cauca y el Eje Cafetero, un patrón que se observa también entre colombianos que migran hacia otros lugares del mundo (OIM, 2012; Guarnizo, 2007; Pardo, 2013). Tampoco es posible afirmar que se trate de mujeres que pertenecen a una sola clase social, puesto que las colombianas en Italia provienen de todos los estratos sociales, si bien la participación de las clases medias es muy consistente (los estratos 3 y 4 acumulan el $68 \%$ del total). Otros elementos de diferenciación entre ellas corresponden al capital humano de que disponen y al capital social, entendido como el que poseen antes de partir pero también como aquel que logran construirse o reconstruirse durante la experiencia migratoria.

Las causas que motivan a las mujeres a emigrar son muchas, y entre ellas, como sostienen Boyd y Grieco (2003), son determinantes algunos factores sistémicos y macroestructurales relacionados con la economía nacional del país de origen y con las dinámicas globales. Igualmente, concurren factores de tipo micro o individuales, algunos derivados de especificidades de género y del ciclo de vida de las familias. Esto lleva a considerar y observar las relaciones de género dentro del contexto familiar, ya que es allí donde generalmente se concretizan las jerarquías y la distribución del poder entre hombres y mujeres. La familia define y asigna papeles diferenciados a sus miembros que de algún modo determinan la motivación y el incentivo para migrar, y al controlar la distribución de los recursos y la información, puede también sostener o, por el contrario, desanimar y hasta coartar la migración.

En el colectivo colombiano en Italia, las causas para emigrar son múltiples, aunque la mayoría $(46,2 \%)$ están ligadas a factores de expulsión, ya que se trata, directa o indirectamente, de causas de tipo económico (tabla 1). Muchas y muchos colombianos sufren en su país por la falta de oportunidades profesionales, la precariedad del trabajo y las condiciones contractuales, causas de insatisfacción que, alimentadas por imaginarios de bienestar en el exterior, se convierten en razones que los motivan a dejar el territorio nacional. Cabe anotar que en Colombia las mujeres, respecto a los hombres, aunque posean las mismas competencias o habilidades, frecuentemente reciben salarios más bajos, y tienen menos ocasiones de hacer carrera y de llegar a cargos de poder o responsabilidad. Muchas de las colombianas que se dirigen a Italia no son ajenas a tales dinámicas, y parecen, además, estar más expuestas a las condiciones de inseguridad del país y a las diversas formas de violencia, aun intrafamiliar o doméstica ${ }^{3}$. Dichos factores tienen repercusiones importantes en el ámbito

3 Según la encuesta Encuesta Nacional de Demografía y Salud (ends) 2010, (realizada por Profamilia cada 5 años, basada en una muestra de aproximadamente 50.000 hogares ubicados en 258 municipios del país), en Colombia existe una fuerte violencia intrafamiliar contra las mujeres: el $65 \%$ de las encuestadas afirman que han sido víctimas 
laboral, productivo y económico de las mujeres, e innegablemente, en los mismos grupos familiares. Además de estas desigualdades de género presentes en la fase premigratoria, hay que considerar las disparidades de clase y, en parte, de procedencia regional, que determinan diferencias sustanciales entre las mujeres migrantes, delineando una multiplicidad de situaciones y experiencias distintas entre sí.

TABLA 1

PRINCIPALES MOTIVOS PARA EMIGRAR DE COLOMBIA SEGÚN EL SEXO (POR RESPUESTAS)

\begin{tabular}{|c|c|c|c|c|}
\hline & Hombres (\%) & Mujeres & Total respuestas (\%) & Total casos $(\%)$ \\
\hline Motivos económicos & 41,0 & 48,9 & 46,2 & \\
\hline $\begin{array}{l}\text { - Falta de oportunidades económicas, profesio- } \\
\text { nales y laborales }\end{array}$ & 31,0 & 31,9 & 31,6 & 60,7 \\
\hline - Ayudar económicamente a la familia & 10,0 & 17,0 & 14,6 & 28,0 \\
\hline Motivos familiares & 12,0 & 14,4 & 13,5 & \\
\hline - Reunirse con familiares ya residentes en Italia & 11,0 & 9,6 & 10,0 & 13,3 \\
\hline - Fracaso sentimental & 1,0 & 4,8 & 3,5 & 6,7 \\
\hline Estudio & 18,0 & 10,7 & 13,2 & 25,3 \\
\hline Aventura & 15,0 & 9,6 & 11,5 & 22,0 \\
\hline Conflictos en Colombia & 8,0 & 10,1 & 9,4 & \\
\hline - Inseguridad & 4,0 & 7,4 & 6,3 & 12,0 \\
\hline - Huir de la violencia o de persecuciones & 4,0 & 2,7 & 3,1 & 6,0 \\
\hline Otras & 6,0 & 6,3 & 6,2 & 12,0 \\
\hline Total & 100,0 & 100,0 & 100,0 & 192,0 \\
\hline Número de respuestas & 100 & 188 & 288 & \\
\hline $\mathrm{N}$ & 53 & 97 & & 150 \\
\hline
\end{tabular}

Fuente: elaboración propia con base en Ciurlo (2013).

No obstante la complejidad del fenómeno, uno de los puntos en común a muchas de las colombianas en Italia, especialmente de los estratos medios y bajos, es que los diversos proyectos migratorios, aun cuando hayan sido decididos de modo autónomo, se sitúan en el marco de estructuras que trascienden la dimensión individual, y se inscriben en todo caso en el marco de las estructuras familiares. Al respecto, Phizacklea (1983) hace notar que la ideología imperante acerca de la maternidad y el compromiso que tienen las mujeres respecto de mantener sus grupos domésticos, la lealtad hacia la familia y la fidelidad filial, hacen que las mujeres asuman el proyecto migratorio como un proyecto grupal y que su trabajo sea consi- 
derado una contribución al proyecto familiar. De hecho, entre las colombianas, usualmente la familia determina la decisión de emigrar y el proyecto migratorio se consolida como una búsqueda para mejorar las condiciones de vida de todo el núcleo doméstico. Muchas de ellas conciben su propio bienestar a partir del bienestar colectivo, y casi nunca desde una óptica exclusivamente personal.

Entre la variedad de casos que se presentan, suscitan una cierta sorpresa las mujeres jóvenes que emigran en el momento del ciclo de vida que coincide con el abandono del núcleo de pertenencia o familia de origen ( $43 \%$ ). Para algunas de ellas (22\%), a diferencia de los hombres ( $0 \%)$, los proyectos migratorios tienen entre sus objetivos el poder contribuir al bienestar de los progenitores -las madres, especialmente-, hermanos, hermanas, sobrinos y otros miembros de la familia extensa.

Se observan también muchos casos de mujeres que tienen a su cargo, directamente, la responsabilidad del sustento familiar o de algunos de sus miembros. Entre ellas están las mujeres cabeza de familia ${ }^{4}(14,4 \%)$, y también mujeres jóvenes que emigran sin tener una pareja estable, compañero o marido, o un propio núcleo familiar formado $(9,3 \%)$, pero con hijos a su cargo y que se apoyan en la familia de origen. La mayor parte de las veces, los hijos se convierten en el motor de la migración y la justificación para partir. Sin embargo, como emerge de varios estudios (Herrera, 2002; Lagomarsino, 2006; Parreñas, 2010; Pedone, 2008; Wagner, 2008), es usual que, entre las diferentes dificultades, también tengan que afrontar la estigmatización y los prejuicios de que son víctimas ${ }^{5}$ por el hecho de no poder realizar el trabajo de reproducción social de modo presencial como consecuencia de la migración. Pero, más allá de la problemática de dichas mujeres, el dato evidencia la difusión del "madresolterismo" al que refiere Pachón (2007), del embarazo adolescente y de las familias monoparentales en Colombia, responsabilidad que recae sobre todo en las mujeres. $Y$ hace ver, además, cuán extenso y radicado está el fenómeno de la irresponsabilidad de los hombres respecto a la prole y hacia su familia en general, así como el machismo y la escasez de respuestas a nivel institucional y de la sociedad respecto de la situación de las mujeres solas. Una realidad que, como explica Gutiérrez de Pineda (1997), se remonta a la época de la Colonia.

de violencia verbal por parte de sus esposos o compañeros; el $37 \%$ de violencia física y el $18 \%$ han sido tocadas o manoseadas sin su consentimiento. Más de un cuarto del total de las mujeres casadas o en unión libre $(28 \%)$ tienen planes de separarse: las causas principales están relacionadas con la violencia, la infidelidad, el incumplimiento de los deberes familiares y la embriaguez de sus parejas. El porcentaje de mujeres maltratadas físicamente-también por otros miembros de la familia- que no denuncian las violencias es casi de tres cuartos del total (73\%) (Profamilia 2010, Cap. Decimotercero).

4 Los datos del Censo de 2005 no reportan información sobre las mujeres cabeza de familia, sin embargo, la encuesta ENDs 2010 ilustra que, en su muestra, los hogares con mujeres jefas de hogar representan el $34 \%$ del total (Profamilia 2010, Cap. Tercero).

5 Se las acusa de ser malas madres o madres que abandonan, que destruyen o desarticulan las familias. 
Otras mujeres que emigran en el marco de proyectos familiares son aquellas que deciden junto con sus maridos $(9,3 \%)$-casi siempre se trata de relaciones formalizadas- viajar solas, asumiendo ellas en primera persona el rol productivo del núcleo familiar.

Para algunas colombianas sin un compromiso familiar estable ( $25 \%$ aprox.), la migración, en cambio, puede significar una búsqueda más personal, generalmente de orden emotivo, luego de la ruptura de una relación afectiva o después de haber vivido situaciones difíciles en esta dimensión vital. Por supuesto, también hay mujeres que planean su viaje con mayor libertad respecto de la familia, con un modelo que se asemeja más al de la mayoría de hombres colombianos en Italia y presentes también en otros países. Un modelo, como sostienen Puyana, Micolta y Palacio (2013, p. 119), caracterizado por menos condicionamientos familiares y sociales, y que persigue objetivos individuales, como por ejemplo, aumentar el capital humano, la promoción profesional, o simplemente el deseo de aventura (tabla 2).

TABLA 2

COMPOSICIÓN DEL HOGAR ANTES DE EMIGRAR SEGÚN EL SEXO

\begin{tabular}{|l|c|c|c|}
\hline & Hombres (\%) & Mujeres (\%) & Total (\%) \\
\hline Núcleo de origen & 56,6 & 43,3 & 48,0 \\
\hline Núcleo origen más hijos & 0,0 & 9,3 & 6,0 \\
\hline Pareja o expareja & 11,3 & 4,1 & 6,7 \\
\hline Pareja + hijos & 11,3 & 9,3 & 10,0 \\
\hline Solo/a + hijos & 3,8 & 14,4 & 10,7 \\
\hline Solo/a & 9,4 & 10,3 & 10,0 \\
\hline Amigos & 1,9 & 10,3 & 4,0 \\
\hline Varios parientes & 5,7 & 3,1 & 4,0 \\
\hline No responde & 0,0 & 1,0 & 0,7 \\
\hline Total & 100,0 & 100,0 & 100,0 \\
\hline N & 53 & 97 & 150 \\
\hline
\end{tabular}

Fuente: elaboración propia con base en Ciurlo (2013).

\section{LA ARTICULACIÓN DE LAS FUNCIONES PRODUCTIVA Y REPRODUCTIVA EN LAS FAMILIAS TRANSNACIONALES}

Para analizar cómo se modifican las relaciones de género durante la migración, resulta útil observar la manera en que impacta la migra- ción misma en la familia y en la distribución de los distintos roles entre sus miembros. Según Lagomarsino (2006, p. 185), las familias no se modifican de manera homogénea ya que son diversas las variables que intervienen en tal proceso: el lugar de donde se parte, el rol del migrante dentro de la familia, el sexo y la 
edad, el tipo de migración, su duración y las condiciones en las que se realiza. A pesar de las diferencias, la migración inevitablemente implica cambios estructurales y en el funcionamiento de los grupos familiares. Cabe recordar, como sugiere Saraceno (1996, p. 243), que la familia en sí no es una realidad estática sino una realidad en movimiento, con cambios que se suceden durante su ciclo de vida; las familias, en el contexto migratorio, suelen tener cambios más bruscos y consistentes, que además estimulan transformaciones y variaciones en los roles tradicionales. Como sostienen Tienda y Booth (1991), se trata, sin embargo, de cambios contradictorios que no van en una sola dirección, disipando las asimetrías preexistentes, sino que estas se reestructuran y redibujan con diversas morfologías.

En el proceso migratorio, generalmente la familia queda encuadrada en dinámicas que involucran el contexto de origen y el de llegada, articulándose frecuentemente como unidades transnacionales que Bryceson y Vourela (2002, p. 3) definen como aquellas familias cuyos miembros viven en espacios geográficos separados durante periodos de tiempo más o menos prolongados, y a veces de modo definitivo. No obstante la distancia que los separa, sus componentes son capaces de mantener vínculos y lazos entre ellos que les permiten seguir sintiéndose parte de una unidad y percibir el propio bienestar en una dimensión colectiva. De ese modo, las familias se conciben a partir de sus dinámicas de negociación y reconfiguración constante, a través de su capacidad de adaptación en el tiempo y en el espacio. Se trata de unidades multisituadas, con residencia y hogar en más de una nación.
Algunas familias colombianas, en la tentativa de articular sus funciones productiva y de reproducción social en el contexto transnacional, deciden que emigren padres o madres dejando a sus parejas o a sus hijos en el país de origen. A nivel de la organización familiar, ello comporta, como sostienen Puyana, Micolta y Palacio (2013, p. 145), que el hogar se desterritorialice y asuma una connotación "glocal", convirtiéndose en un hogar que funciona por encima de las fronteras, estructurado entre la dimensión local y la dimensión global, en el que circula un abundante flujo tanto de recursos económicos y materiales como de cuidados (Merla, 2014).

En el colectivo colombiano presente en Italia, cerca de un quinto de las unidades familiares son de este tipo. Se trata sobre todo de familias en las que quienes emigran son las mujeres (23\% frente a $15,1 \%$ de los hombres), asumiendo, como se vio con anterioridad, el rol productivo dentro de la familia. Son jefas de hogar, o bien miembros de una familia nuclear en la que el marido se queda con los hijos en Colombia. Sin embargo, también hay familias, aunque en menor cantidad, en las que el hombre es quien emigra, dejando a la pareja y la prole en Colombia. En este caso, los cambios en la organización del hogar no son muy relevantes, pues la labor de cuidado de los hijos la sigue realizando la pareja o la expareja, y en una escasa proporción pasa a otras mujeres de la familia, lo que garantiza una cierta continuidad en la vida cotidiana de los hijos. Los hombres migrantes continúan con su rol productivo, se involucran poco en las decisiones que atañen a los hijos en Colombia y se preocupan, en cambio, por mantener las 
relaciones afectivas a distancia mediante los diversos medios de comunicación que tienen a disposición.

Cuando son ellas las que emigran, los cambios son mayores: con frecuencia el cuidado de los hijos pasa a manos de otras mujeres, en general parientes por la línea materna-casi siempre hermanas o abuelas-, o, en pocos casos, a mujeres externas al grupo. Con estas personas establecen relaciones informales de reciprocidad, si bien en ocasiones pagan por sus servicios, especialmente en los estratos más altos, donde es muy frecuente recurrir a empleadas domésticas. No es habitual que los hombres se hagan cargo del cuidado de los hijos, y ello aun en los casos en que no son la principal fuente de sostén de la familia. Son las redes de apoyo -constituidas en gran parte por mujeres-, con los recursos que proporcionan, las que permiten reorganizar las tareas en el hogar. De esta manera, las migrantes pueden partir, pero tienen el poder de no poner en discusión los roles de género y una división más equitativa de las diferentes tareas entre las parejas.

A pesar de que las mujeres asumen las responsabilidades económicas del grupo, y de hallarse fuera del país, siguen participando activamente en el rol materno y reproductivo, junto con las encargadas del cuidado de la prole en Colombia. Utilizan todos los medios a su disposición para suplir la ausencia y continuar con esta tarea a distancia, que algunas viven de manera conflictiva. Cerca de dos tercios (69,6\%) experimentan un fuerte sentimiento de culpa por dejar a sus hijos, cuatro quintos $(78,3)$ sienten ansiedad por no verlos crecer ni poder estar a su lado, pero también muchas $(73,9 \%)$ se enorgullecen del sacrificio que hacen para brindarles un mejor futuro. Sobre ellas pesan significativamente los prejuicios de no ser madres presenciales y las representaciones tradicionales de la maternidad habituales en Colombia como también en Italia, lo que alimenta y reproduce en las propias mujeres una identidad de género de tipo patriarcal. Esta parece influir, junto con otros factores de tipo emotivo, en la propensión que tienen las colombianas más que los colombianos, a reunirse con la prole; proceso que por motivos burocráticos y económicos ${ }^{6}$, y por la dificultad para conciliar el trabajo productivo y el reproductivo una vez que los hijos están en Italia, puede llegar a durar muchos ańos.

En la asignación de roles dentro de la familia transnacional, se van delineando las diversas posiciones que ocupan las mujeres durante el proceso migratorio y las desigualdades respecto a los hombres. No es suficiente, sin embargo, saber que las mujeres asumen el rol productivo, adquiriendo en ocasiones cuotas de poder por el hecho de manejar los recursos

6 Para la reunificación familiar es necesario disponer de una renta anual no inferior al subsidio social anual (€5.818,93 en 2014), incrementado en la mitad para cada familiar que se quiere reunir. Se pueden reunir: la pareja mayor de edad que no esté separada legalmente, los hijos menores de edad no casados; los hijos mayores de edad que estén a cargo del interesado y que sean inválidos totales; los padres a cargo (de más de 65 ańos) cuando no haya otros hijos en el país de proveniencia que puedan hacerse cargo o estén impedidos para hacerlo por graves problemas de salud. 
económicos de la familia; es también relevante conocer, como sostienen Oso y Catarino (1997), el lugar que ocupaban la mujeres en el sistema de producción en el país de origen y el tipo de inserción laboral en el país de destino.

En este sentido, se observa que estos dos aspectos están relacionados entre sí, ya que la inserción de las mujeres en la sociedad italiana está supeditada en parte a las condiciones que estas tenían en Colombia. De hecho, para quienes parten en medio de circunstancias de elevada vulnerabilidad y precariedad, estas tienden a perdurar en el nuevo contexto. Las mujeres con menores recursos, ya sea de tipo económico o social, al igual que aquellas que tienen una carga de responsabilidades mayor, se ven penalizadas en su proceso de inserción laboral en Italia. Esto es perfectamente evidente entre las mujeres solas con hijos a cargo, jefas de hogar. Algunas de ellas, frente a la presión que comporta tener que sostener al grupo familiar, no encuentran otra opción que trabajar en el servicio doméstico o en las labores de cuidado a la persona ${ }^{7}$, aun sin desearlo: téngase en cuenta que sobre el trabajo doméstico pesan en Colombia fuertes estigmatizaciones de clase. Es usual, además, que tengan que aceptar condiciones laborales inestables e informales, con una vida social muy limitada como ilustra
Parreñas (2005). Algunas veces, el aislamiento comporta el que no conozcan sus derechos, que, por tanto, no logren ejercitarlos, y que no perciban muy claramente las discriminaciones que sufren por ser inmigrantes, confundiéndolas con comportamientos discriminatorios sobre la base del género ${ }^{8}$, a los que ya estaban expuestas en Colombia, por lo que los consideran, de algún modo, "normales".

No obstante la frustración, en algunos casos, por las condiciones antes mencionadas, en general muchas mujeres (75,3\%) están medianamente satisfechas por el estilo de vida que tienen en Italia. En ello contribuye el que el ingreso económico con que cuentan les permite lograr los objetivos de su proyecto migratorio y cumplir con el compromiso familiar -en especial con sus hijos-, elemento central de su vida como inmigrantes. Se nota igualmente una cierta satisfacción entre muchas mujeres que logran reunirse con sus hijos en Italia, ante una forma de vida que les permite disfrutar de servicios sociales que resultan fundamentales para hacer frente a la educación de la prole con costos bajos y con niveles de calidad aceptables. Este elemento demuestra ulteriormente el apego a una identidad de género que las muestra como protagonistas de las labores reproductivas.

\footnotetext{
7 Ambrosini (2005, p. 69) ilustra cómo el mercado laboral italiano está fuertemente segmentado, y cómo el sector de los servicios domésticos y de cuidado de la persona se consolida como el nicho laboral más común entre las mujeres extranjeras, debido a la externalización de tales servicios fuera de la familia.

8 Ello parece confirmarlo la encuesta del Istituto Nazionale di Statistica (ISTAT) hecha entre la población italiana, en la que emerge claramente que gran parte de los ciudadanos y ciudadanas considera que sobre las mujeres en Italia pesan estereotipos y discriminaciones de género que dictan fuertes desigualdades en diversos ámbitos, como el laboral (ISTAT, 2013).
} 


\section{LA CONSTRUCCIÓN SOCIAL DE LO FEMENINO EN EL PROCESO MIGRATORIO}

Además de las mujeres madres de familia que emigran, una gran parte de las colombianas parten cuando no han formado aún su propio hogar y viven en su núcleo de origen (52,6\%). El viaje coincide con un momento de cambio fisiológico del grupo familiar, y, por tanto, la migración tiene menores consecuencias en la organización práctica del hogar y la estructura del grupo. Hay que considerar, sin embargo, que en algunos casos se trata de mujeres que ya tienen hijos, pero viven con la familia de origen o la familia extensa, apoyándose en ella para poder afrontar las diversas responsabilidades.

Si bien es cierto que en este tipo de formas familiares, y cuando son los hijos los que emigran, sus miembros no pretenden articular las funciones reproductivas y productivas del grupo, no se puede negar su carácter transnacional. Como sostiene Merla (2014, p. 92), las familias transnacionales son extremadamente variadas y las relaciones diádicas madre/padrehijo no son las únicas que generan un intercambio de bienes materiales e inmateriales a través de las fronteras. Las familias transnacionales están constituidas también por la relación con otros miembros de la parentela e implican redes mucho más amplias. Son importantes no solo por los recursos materiales que ofrecen a quien se queda y a quien se va, sino también por el intercambio de carácter simbólico. De hecho, Basch et al. (1994, p. 79) afirman que las familias, siendo depositarias de prácticas e ideologías culturales, influyen en la identidad de sus miembros no solo antes de partir, sino también sucesivamente, en el nuevo contexto.
En este sentido, son también un vehículo de las relaciones de género a través de los confines.

Para casi la mayoría de colombianas en Italia $(85,5 \%)$, la familia en su patria y las relaciones transnacionales que se mantienen con una comunicación rica y constante, representan un apoyo fundamental para hacer frente a dificultades que deben afrontar en el nuevo contexto. Así por ejemplo, frente a la nostalgia, la soledad, la burocracia, las contrariedades por tener que asumir costumbres diferentes, la falta de reconocimiento de los títulos de estudio y los procesos de descalificación laboral y social, o las diferentes formas de discriminación; en últimas también frente a una integración por lo bajo en la sociedad italiana, que Ambrosini (2001) denomina subalterna, las familias son una fuente de bienestar psíquico y emocional muy importante.

Son, asimismo, una manera de mantener la pertenencia a la tierra y la cultura de origen para casi todas las mujeres (93,8\%), y una forma para mantener vivo aquello que, desde el sistema clasificatorio de género, se expresa en las representaciones sociales de lo femenino. Como sugiere Jodelet (1986, pp. 469 y ss.), se trata de representaciones simbólicas que dan atribuciones a la conducta subjetiva y objetiva de las personas. Desde una perspectiva de género, en la búsqueda de construir nuevas subjetividades femeninas, a un quinto de las migrantes $(26,8 \%)$ las ayuda el estar en Italia, lejos de su familia de origen, pues sienten menos presión y control de su grupo familiar que estando en Colombia. Sin embargo, la familia en Colombia y las relaciones con algunos familiares, lejos de desaparecer, siguen presentes, demandando frecuentemente formas de lealtad. 
Por el contrario, en lo que respecta a quien migra solo, es usual construirse una vida afectiva en el nuevo contexto, ello a pesar de los frenos que la misma migración impone. Entre las mujeres colombianas se nota una mayor propensión y facilidad a formar su propia familia durante la migración, respecto a los hombres colombianos, haciéndolo además, con frecuencia, con una pareja italiana. Ello sucede no solo entre quienes vivían con su familia de origen, sino también en algunos casos de mujeres que tenían a sus espaldas situaciones más complejas de separaciones, divorcios, con hijos a cargo, que generalmente buscan relacionarse con un tipo de identidad masculina diferente. Algunas aspiran, de hecho, a tener una relación estable con una persona responsable y con quien sea posible construir un proyecto de vida común basado en criterios de seguridad y estabilidad.

Entre los hombres no es tan frecuente construir una familia en Italia y, menos aún, escoger una pareja italiana, ya que este tipo de elección representa solo una pequeña parte. Tal comportamiento es habitual, en general, entre los inmigrantes en Italia ${ }^{9}$ y también entre los colombianos en otros países europeos; Posso Quinceno (2007, pp. 55-57), para el caso espańol, evidencia la misma tendencia. Ello, según la autora, depende en parte del hecho de que los hombres colombianos parecen tener más dificultades en adaptarse a costumbres diferentes y a una cultura donde la mujer ha alcanzado un mayor grado de emancipación, lo que genera obstáculos a la hora de intentar establecer relaciones afectivas con ellas (tabla 3).

TABLA 3

ESTADO CIVIL SEGÚN EL SEXO Y LA NACIONALIDAD DE LA PAREJA

\begin{tabular}{|c|c|c|c|c|c|c|c|c|c|c|c|}
\hline \multirow[t]{2}{*}{ Estado civil } & \multicolumn{2}{|c|}{ Sin pareja } & \multicolumn{2}{|c|}{$\begin{array}{c}\text { Nacionalidad } \\
\text { colombiana }\end{array}$} & \multicolumn{2}{|c|}{$\begin{array}{c}\text { Nacionalidad } \\
\text { italiana }\end{array}$} & \multicolumn{2}{|c|}{$\begin{array}{c}\text { Otra } \\
\text { nacionalidad }\end{array}$} & \multicolumn{2}{|c|}{ Total } & \multirow{2}{*}{$\begin{array}{l}\text { Total } \\
\% \mathrm{H}+\mathrm{M}\end{array}$} \\
\hline & H (\%) & M (\%) & H (\%) & M (\%) & H (\%) & M (\%) & H (\%) & M (\%) & H (\%) & M (\%) & \\
\hline Soltero/a & 95,0 & 100,0 & 0,0 & 0,0 & 0,0 & 2,4 & 0,0 & 80,0 & 35,8 & 27,8 & 30,7 \\
\hline Casado/a & 0,0 & 0,0 & 50,0 & 37,5 & 50,0 & 65,9 & 40,0 & 10,0 & 30,2 & 41,2 & 37,3 \\
\hline Unión libre & 0,0 & 0,0 & 27,3 & 20,8 & 50,0 & 17,1 & 60,0 & 0,0 & 22,6 & 13,4 & 16,7 \\
\hline Separado/a & 0,0 & 0,0 & 18,2 & 20,8 & 0,0 & 12,2 & 0,0 & 10,0 & 7,5 & 11,3 & 10,0 \\
\hline Divorciado/a & 5,0 & 0,0 & 4,5 & 16,7 & 0,0 & 0,0 & 0,0 & 0,0 & 3,8 & 4,1 & 4,0 \\
\hline Viudo/a & 0,0 & 0,0 & 0,0 & 4,2 & 0,0 & 2,4 & 0,0 & 0,0 & 0,0 & 2,1 & 1,3 \\
\hline Total columna & 100,0 & 100,0 & 100,0 & 100,0 & 100,0 & 100,0 & 100,0 & 100,0 & 100,0 & 100,0 & 100,0 \\
\hline Total línea & 37,1 & 26,8 & 41,5 & 24,7 & 11,3 & 42,3 & 9,4 & 6,2 & 100,0 & 100,0 & 100,0 \\
\hline $\mathrm{N}$ & 20 & 26 & 22 & 24 & 6 & 41 & 5 & 6 & 53 & 97 & 150 \\
\hline
\end{tabular}

Fuente: elaboración propia con base en Ciurlo (2013).

9 Los datos sobre los matrimonios mixtos en Italia muestran que solo un cuarto de ellos se realizan entre hombres extranjeros y mujeres italianas. En 2012, de las 167 bodas que se celebraron entre parejas colombo-italianas, 162 correspondieron a uniones entre mujeres colombianas y hombres italianos (ISTAT - http://stra-dati.istat.it/). 
La existencia de parejas mixtas entre colombianas e italianos, por un lado, parece indicar una gran facilidad de las colombianas para relacionarse e integrarse en el nuevo contexto, y por otro, también, de alguna manera, el que ellas están ancladas a la representación social de la mujer débil que necesita de una pareja a su lado para sobrevivir. En algunos casos, parece pesar el que en Colombia sea usual, en algunos contextos, considerar que tener una pareja extranjera puede ofrecer mejores condiciones de vida, al igual que un cierto estatus y prestigio social.

Cabe mencionar que algunas colombianas ya antes de emigrar conocían a su pareja italiana, lo que lleva a pensar que existe un mercado matrimonial del que las mujeres con hijos a cargo, una edad avanzada y menos libertad de movimiento, quedan excluidas. Según Hurtado Saa (2008, p. 350), en Italia existe dicho fenómeno, que demanda mujeres extranjeras, especialmente aquellas con características físicas y culturales diferentes de las europeas. Lo anterior revela la existencia de algunos estereotipos en el contexto italiano, referidos a que las extranjeras, en especial las latinoamericanas, serían menos exigentes, más "sumisas” y “exóticas"; estereotipos presentes de igual modo en otros contextos europeos (Beday Hauser y Desboilles, 2007; Cepal, 2003).
Pero más allá de las causas por las que se forman dichas familias, es indudable que tener pareja italiana facilita la incorporación en el contexto de llegada. Por ejemplo, en lo que se refiere a los trámites de ingreso a Italia y posteriormente -en especial si la relación está formalizada- se convierte en el motivo por el que se obtiene un estatus migratorio que permite vivir regularmente y de modo estable en el nuevo país ${ }^{10}$. De la misma manera, facilita los procesos de reunificación familiar y hace posible la adquisición de la ciudadanía italiana ${ }^{11}$, que a su vez permite participar en las elecciones y acceder al empleo público, del que por ley están excluidos los ciudadanos extranjeros. En muchos casos -aunque no siempre- ofrece un capital social que puede traducirse en otras formas de capital.

Al observar los procesos de autonomía de las mujeres, en lo que concierne específicamente al ámbito laboral, se advierte que aquellas sobre cuyas espaldas recae un menor peso económico, que tienen más flexibilidad para afrontar las necesidades monetarias, que son más jóvenes o que cuentan con un capital social más consistente, logran insertarse en sectores laborales diferentes del doméstico. Generalmente, no se trata de trabajos que tienen en cuenta los estudios que han realizado, y se

10 Para poder residir en Italia regularmente, es necesario tener: el Permesso di soggiorno (Permiso de estadía) o la Carta di soggiorno (Tarjeta de estadía). El primero es otorgado por diversos motivos, entre ellos por motivos familiares, y tiene una duración que va de tres meses a dos años. El segundo, es un permiso por tiempo indeterminado y puede solicitarse después de cinco años de residencia regular no interrumpida o bien por matrimonio con ciudadano/a italiano/a o perteneciente a la comunidad europea.

11 La Ley de Ciudadanía (Ley 91 de 1992) se basa en dos principios: el derecho a la transmisibilidad por descendencia (ius sanguinis), y la concesión, que puede ser por matrimonio con ciudadano/a italiano/a (es un derecho) y por residencia en el territorio nacional después de diez años de residencia regular (a discreción de las autoridades). 
hallan casi siempre en el ámbito de los servicios, pero al menos por fuera de aquel sector laboral. Entre las mujeres colombianas se nota, asimismo, un fuerte dinamismo y espíritu empresarial, como también una fuerte resiliencia. Más que los hombres, desarrollan procesos de movilidad ascendente para salir de los nichos laborales clásicos destinados a los inmigrantes en Italia (CNEL, 2012), no siempre siguiendo recorridos fáciles. Algunas mujeres recurren al trabajo autónomo o al autoempleo -especialmente aquellas que tienen pareja italiana- para lograr mayores satisfacciones laborales y un más elevado reconocimiento social, aunque es frecuente que tengan menos seguridades de tipo económico y contractuales (tabla 4).

TABLA 4

EMPLEO EN ITALIA SEGÚN EL SEXO

\begin{tabular}{|l|c|c|c|}
\hline & Hombres (\%) & Mujeres (\%) & Total (\%) \\
\hline Estudiante & 11,3 & 5,2 & 7,3 \\
\hline Desempleado/a - Hogar & 3,8 & 12,4 & 9,3 \\
\hline Trabajo doméstico y cuidado a la persona & 7,5 & 26,8 & 20,0 \\
\hline Empleado/a en: servicios, producción y comercio & 52,8 & 23,7 & 34,0 \\
\hline Trabajador/a autónomo/a & 7,5 & 12,4 & 10,7 \\
\hline Por cuenta propia (informal) & 3,8 & 10,3 & 8,0 \\
\hline Otro & 13,2 & 9,3 & 10,7 \\
\hline Total & 100,0 & 100,0 & 100,0 \\
\hline N & 53 & 97 & 150 \\
\hline
\end{tabular}

Fuente: elaboración propia con base en Ciurlo (2013).

Las evidencias empíricas muestran así que la posibilidad de que las mujeres activen dichas trayectorias depende en gran parte de las condiciones de vida que tienen, también familiares (que pueden variar con el tiempo), del capital social, en parte de las capacidades personales e, infortunadamente, solo en una mínima parte, del capital humano que poseen. Para las mujeres, acceder a trabajos más gratos representa una fuente de satisfacción enorme; sin embargo, no siempre significa por sí mismo superar las brechas de género imperantes. A este propósito, cabe señalar que en Italia la condición de la mujer en general no es muy alentadora. Existen diversos indicadores que revelan un trato poco paritario entre hombres y mujeres, lo que se refleja, entre otras cosas, en la división de los roles dentro de la familia, en un mercado laboral al que tienen un acceso limitado las mujeres -en especial si tienen hijos y otros parientes que cuidar-y en el que, además, son remuneradas con salarios más bajos y tienen menores posibilidades de alcanzar cargos de responsabilidad y de progresar profesionalmente (World Economic Forum, 2013). 


\section{CAMBIOS EN LOS ROLES DE GÉNERO EN LAS FAMILIAS}

Aunque con anterioridad se abordó el tema del trabajo reproductivo y productivo en las familias transnacionales, para comprender los cambios que se producen en las mujeres luego de la migración, es importante observar también cómo se dividen algunos roles en las parejas que conviven bajo el mismo techo.

Si se examina la responsabilidad económica, es natural que en los núcleos compuestos por mujeres solas sean ellas, en Italia, las principales proveedoras económicas de sí mismas y del grupo familiar cuando tienen parientes a su cargo (el $72 \%$ de los casos). Cuando se encuentran en pareja, se notan situaciones diferentes que muestran una realidad que no se puede definir como unitaria. Las mujeres en unión libre en Italia, generalmente proveen por sí mismas a su manutención; entre las parejas casadas, algunas de las migrantes que en Colombia tenían trabajo remunerado fuera de la familia, lo siguen ejerciendo en Italia, especialmente si el proyecto migratorio es un proyecto familiar en el que ellas migran en un primer momento. Es de notar, sin embargo, que en las tres cuartas partes de los casos $(64,9 \%)$ se verifica una disminución de la participación de las mujeres en el trabajo productivo, una tendencia que es muy evidente entre quienes están casadas con un italiano. En el tipo de pareja mixta formalizada, muchas colombianas, cuando tienen trabajos mal remunerados, ocasionales y poco estables, prefieren abandonar el mercado laboral y convertirse en amas de casa, acentuando su menor participación en la manutención económica, que depende en gran parte del marido.
Es de notar, como afirma Bonizzoni (2009, p. 44), que a muchas migrantes se les dificulta excesivamente conciliar el trabajo productivo y el reproductivo por la falta de redes de apoyo con las cuales contar, y por los escasos servicios que ofrece el Estado. Estas carencias, junto con la dificultad concreta de encontrar trabajo - especialmente en la actual crisis económica-, influyen de manera notable en su menor participación en el ámbito productivo. Hay que considerar, además, que no todas las mujeres asumen el ser proveedoras como una elección personal, sino que se ven obligadas a serlo por las circunstancias. Según Solé y Parella (2007, p. 15), muchas mujeres migrantes siguen considerando que el modelo ideal de familia es aquel en el que los hombres asumen el papel de sustentadores mientras ellas se ocupan de las tareas de cuidado en el hogar. Por tanto, perciben el trabajo productivo como una imposición que no las hace sentir realizadas y las obliga a enfrentar largas jornadas laborales, además de impedirles ser madres presenciales, que es lo que en realidad desearían.

A pesar de la escasa participación de algunas mujeres en el trabajo productivo en la unidad familiar durante la migración, ello no parece comprometer su capacidad de decisión respecto a la administración de los bienes económicos del hogar. En este sentido, se nota un gran protagonismo de las migrantes y una mayor equidad entre hombres y mujeres (55\% de los hombres frente al $45 \%$ de las mujeres), que expresa formas de empoderamiento ya que en este se prevé el acceso de las mujeres al control de diferentes tipos de recursos, en este caso, en el seno del grupo familiar.

Retornando a la división sexual del trabajo, otro elemento por observar es el trabajo domésti- 
co y de cuidado de los miembros del hogar, que en los cánones más tradicionales se delega a las mujeres. En Colombia esta división está todavía presente. Puyana (2003) destaca, sin embargo, que en los últimos años se han venido verificando cambios que tienen efectos poco homogéneos en las familias. En ello influyen diversas variables, como el estrato social de pertenencia, el capital humano de que disponen los miembros de la familia, la zona geográfica de residencia, el tipo de familia y la manera como el grupo afronta la modernidad. El estudio muestra que alrededor de un tercio de las familias se caracteriza por mantener aún los roles tradicionales, pero cerca de la mitad de las mismas se halla en una etapa de "transición" en la que se notan cambios lentos y a veces contradictorios respecto a la división sexual de los roles.

En la migración también se presentan situaciones bastante heterogéneas y, de hecho, entre las parejas que en Italia han reunido a sus hijos se advierte una cierta participación de los hombres en el trabajo doméstico, aunque no en medida muy relevante (el $37 \%$ aprox.). Esta crece entre las parejas más jóvenes, y de manera notable y equitativa entre quienes, en cambio, no tienen hijos. En los diversos tipos de familia, nucleares o monoparentales, aunque las mujeres tengan una cierta autonomía económica y un rol importante en cuanto a las decisiones acerca de la administración de los bienes y recursos familiares, el mayor peso del trabajo doméstico permanece a su cargo, con algunas variaciones según la edad. En efecto, entre las migrantes más jóvenes los modelos de comportamiento cambian, especialmente gracias a nuevos modelos culturales que circulan a nivel global, y no como efecto de la migración en sí, o por el contacto con el modelo italiano, que también reproduce en parte el modelo patriarcal en el que el hombre es el principal proveedor de las necesidades económicas y las mujeres se dedican a las tareas del hogar (ISTAT, 2013; Lanzoni, 2014; Saraceno, 2005).

En lo que respecta específicamente al cuidado de los hijos, se ve una mayor participación de los hombres, también entre aquellos que en Colombia se desentendían de esta labor. En las parejas que después de procesos de reunificación familiar reconstruyen su hogar en Italia, se evidencia una distribución bastante equitativa del cuidado de los hijos ( $55 \%$ lo hacen las mujeres y $45 \%$ los hombres), ya que en muchos casos es la única manera como la familia suple esta necesidad. Un comportamiento similar está presente también en las parejas mixtas y en las familias donde las mujeres no trabajan, aunque es cierto que muchas migrantes siguen pensando que esta es una labor típicamente femenina que ellas deben realizar. De hecho, Rivas y Gonzálvez (2009, p. 187), observan que entre las migrantes perdura la concepción de la mujer como figura indispensable para la crianza y el crecimiento de los hijos.

Si el cuidado de los hijos corre en buena parte por cuenta de las mujeres, las decisiones sobre ellos se toman de manera paritaria entre madres y padres. Cabe señalar que se trata de una situación que poco cambia respecto a cuando la familia vivía en Colombia. De hecho, ya en el país de origen, aunque las mujeres debían realizar materialmente las tareas en el hogar, esto no implicaba que no tuvieran un peso en la capacidad de decisión y que ello correspondiera únicamente al hombre. En este sentido, pesa el que en Colombia, si bien el 
modelo patriarcal está aún arraigado, especialmente en algunas regiones del país (Gutiérrez de Pineda, 1997), las mujeres tienen poder de decisión en las familias, que deriva no solo del rol que allí ejercen, sino también de las múltiples formas de irresponsabilidad de los varones en el ámbito familiar que dejan inevitablemente un vacío de importancia.

En las familias monoparentales con jefatura de mujeres es usual que los hombres se comprometan poco con el cuidado material de los hijos y también con las decisiones que les atañen. Esto se verifica, como se mencionó, cuando son ellos quienes migran dejando a sus parejas o exparejas al cuidado de los hijos en Colombia. Es cierto también que, en especial cuando los hijos están en Italia, se notan formas de paternidad en las que aumenta la responsabilidad de los hombres, lo que se expresa no solo en su papel decisorio, sino también en su papel activo en los cuidados.

En el contexto transnacional es posible ver el comportamiento desigual entre hombres y mujeres respecto a las responsabilidades también en otros aspectos. Si se observan las remesas económicas que envían las y los migrantes, se puede notar que las mujeres muestran una mayor asiduidad con envíos mensuales en relación con los hombres $(38,1 \%$ frente a $30,2 \%)$, y que en general son mujeres, a su vez, las principales administradoras de tales recursos en Colombia.

En la utilización de las remesas económicas, las migrantes privilegian el que el dinero sea empleado en las necesidades del hogar, como la manutención de la familia y los gastos de educación y salud, lo cual evidencia, aun en la distancia, una mayor implicación de las mujeres en la reproducción social del grupo familiar en Colombia. Los hombres destinan sumas menos consistentes a la reproducción, $\mathrm{y}$ a la vez cifras mayores a formas de ahorro personal, lo que es índice de un menor sentido de corresponsabilidad con el bienestar colectivo de su grupo familiar. No se puede negar, sin embargo, que algunos hombres que emigran con un proyecto familiar demuestran un fuerte compromiso y responsabilidad con quien se queda en Colombia, asumiendo el rol de proveedores principales de la familia, pero reproduciendo un modelo en el que se mantiene prácticamente inalterada la división sexual del trabajo (tabla 5).

TABLA 5

USOS PRINCIPALES DE LAS REMESAS ENVIADAS A COLOMBIA SEGÚN EL SEXO

\begin{tabular}{|l|c|c|c|c|c|c|}
\hline \multirow{2}{*}{} & \multicolumn{2}{|c|}{ Manutención de la familia } & \multicolumn{2}{c|}{ Educación y salud } & \multicolumn{2}{c|}{ Ahorro } \\
\cline { 2 - 7 } & $\mathrm{H}(\%)$ & $\mathrm{M}(\%)$ & $\mathrm{H}(\%)$ & $\mathrm{M}(\%)$ & $\mathrm{H}(\%)$ & $\mathrm{M}(\%)$ \\
\hline No envía remesas & 17,0 & 18,6 & 17,0 & 18,6 & 17,0 & 18,6 \\
\hline Sí & 45,3 & 58,8 & 27,0 & 49,6 & 17,0 & 8,2 \\
\hline No & 34,0 & 20,6 & 52,2 & 29,7 & 60,4 & 71,1 \\
\hline No responde & 3,8 & 2,1 & 3,8 & 2,1 & 5,7 & 2,1 \\
\hline Total & 100,0 & 100,0 & 100,0 & 100,0 & 100,0 & 100,0 \\
\hline N & 53 & 97 & 53 & 97 & 53 & 97 \\
\hline
\end{tabular}

Fuente: elaboración propia con base en Ciurlo (2013). 
En la vida transnacional, no se puede hacer caso omiso de la participación activa de las mujeres en las diferentes redes de apoyo que se dan durante la migración. Estas son las que en gran parte determinan la llegada de colombianos a Italia (en el 50,5\% de los casos), y resultan fundamentales en el primer momento de su arribo. Son esenciales también para que muchas mujeres puedan emigrar supliendo el trabajo de cuidado en Colombia, pero influyen de modo consistente en su movilidad, encuadrándola en un sistema de obligaciones y expectativas de carácter y alcance normativo. Con frecuencia, reafirman los estereotipos patriarcales, asignando roles y responsabilidades a mujeres y hombres, atribuyendo derechos y ofreciendo oportunidades, y estructurando en parte las relaciones de género.

\section{A MODO DE CONCLUSIÓN}

Los procesos de emancipación de las mujeres migrantes son complejos y a menudo ambivalentes, lo que evidencia ganancias y pérdidas en los contextos en que se hallan involucradas, y en donde juega un papel importante la intersección de desventajas en razón de las diversas posiciones entre las mismas mujeres. Estas derivan de las responsabilidades que pesan sobre sus hombros, de sus condiciones socioeconómicas y familiares antes de partir y también en el nuevo contexto.

Como se pudo observar, no es suficiente cambiar los roles dentro de las familias para modificar el estatus y la posición de las mujeres $\mathrm{y}$, más ampliamente, transformar las relaciones de género. De hecho, estas permanecen inalteradas en muchos casos, aunque las mujeres cuenten con un trabajo remunerado, devenguen un sueldo y en algunas ocasiones sean las proveedoras principales del hogar. Téngase en cuenta, además, que en el nuevo contexto algunas parecen percibir menos el control familiar. El hecho de que dentro de la familia las categorías de la construcción social de las desigualdades entre hombres y mujeres estén estrechamente relacionadas con las dimensiones afectiva, emotiva y sexual del individuo, hace que sea aún más difícil cambiar las estructuras dominantes, convirtiendo además a la familia en un vehículo que no solo transmite sino que consolida las ideologías de género tradicionales.

A pesar de la potencia conservadora de la familia, los hallazgos ponen de manifiesto también la capacidad de agencia de muchas mujeres, que logran aprovechar algunas ventajas que tienen a su favor, no obstante las múltiples dificultades que deben afrontar. De hecho, mediante la migración, y también gracias a su experiencia transnacional y a su rol activo en las redes, ciertas mujeres logran construir nuevas subjetividades femeninas que evidencian el potencial emancipador de diferentes factores, o, como sugiere Bonizzoni (2009, p. 44), en la combinación de varios recursos socioeconómicos se van delineando complejos y no siempre lineales procesos de empoderamiento.

\section{REFERENCIAS}

Anthias, F. (2013). Intersectional what? Social divisions, intersectionality and levels of analysis. Ethnicities, 13 (1), 3-19.

Anthias, F. (2000). Metaphors of home: Gendering new migrations to southern Europe. En Anthias, F. 
y Lazaridis, G. (eds.). Gender and migration in southern Europe. Women on the move (pp. 15-48). Oxford: Berg.

Anthias, F. y Yuval Davis, N. (1983). Contextualizing feminism. Gender, ethnic and class divisions. Feminist Review, 15, 62-75.

Ambrosini, M. (2001). La fatica di integrarsi. Bologna: Il Mulino.

Ambrosini, M. (2005). Sociologia delle migrazioni. Bolonia: Il Mulino.

Basch, L., Glick Shiller, N. y Szanton Blanc, L. (1994). Nations unbound: Transnational Projects, postcolonial predicaments and deterritorialized nationstates. New York: Gordon and Breach.

Beday Hauser, P. y Desboilles, M. L. (2007). De l'exotique au familiar: copules mixtes latino-suisses. En Bolzman, C., Carbajal, M. y Mainardi, G. (eds.). La suisse au rytme latino. Dinamiques migratoires del Latino-Américains: logiques d'action, vie quotidienne, pistes d'interventions dans les domaines du social et de la santé (pp. 227-246). Ginebra: Les Editions.

Bonizzoni, P. (2009). Famiglie globali. Le frontiere della maternità. Torino: UTET Università.

Bonizzoni, P. y Boccagni, P. (2013). Care (and) circulation revisited. En Baldassar, L. y Merla, L. (eds.). Transnational families, migration and the circulation of care: Understanding mobility and absence in family life. New York y Abingdon: Routledge.

Boyd, M. y Grieco, E. (2003). Women and migration: Incorporating gender into international migration theory. Migration Information Source. Recuperado de http://www.migrationinformation.org/ Feature/display.cfm?id=106

Brah, A. (2004). Diferencia, diversidad, diferenciación. En VV.AA. Otras inapropiables. Feminismos desde las fronteras (pp. 107-136). Madrid: Traficantes de Sueños.
Bryceson, D. y Vourela, U. (Eds.) (2002). The transnational family. New European frontiers and global networks. London - New York: Berg.

Campani, G. (2000). Genere, etnia e classe. Migrazioni femminili tra esclusione ed identità. Pisa: ETS.

Carling, J. (2005). Gender dimensions of international migration. Global Migration Perspectives, 35. Ginebra: Global Commission on International Migration.

Catarino, C. y Morokvasic, M. (2005). Femmes, genre, migration et mobilités. Revue européenne des migrations internationales, 21(1). Recuperado de http://remi.revues.org/2534

CEPAL (2003). La trata de mujeres: sus conexiones y desconexiones con la migración y los derechos humanos. Serie Población y Desarrollo, 39, 5-33.

Ciurlo, A. (2014). El colectivo colombiano en Italia y el impacto de la cuestión migratoria en las relaciones internacionales entre los dos países. Revista Internacional de Cooperación y Desarrollo, 1(2), 125-162.

Ciurlo, A. (2014). Género y familia transnacional. Un enfoque teórico para aproximarse a los estudios migratorios. Rev. Cient. Gen. José María Córdova, 12(13), 127-161.

Ciurlo, A. (2013). Migración colombiana hacia Italia a la luz del género y de la familia transnacional. Bogotá: Universidad Antonio Nariño - Fundación Esperanza.

Crenshaw, K. (1991). Mapping the margins: Intersectionality, identity politics, and violence against Women of Color. Stanford Law Review, 43(6), 1241-1299.

CNEL (2012). Il ruolo degli immigrati nel mercato di lavoro italiano. Documento de Trabajo (CNEL Consiglio dell'Economia e del Lavoro - Ministero del Lavoro e delle Politiche Sociali). 
Gregorio Gil, C. (1998). Migración femenina. Su impacto en las relaciones de género. Madrid: Narcea.

Gregorio Gil, C. (2009). Mujeres inmigrantes: colonizzando sus cuerpos mediante fronteras procreativas, etnico-culturales, sexuales y reproductivas. Viento Sur, 104, 42-54.

Guarnizo, L. E. (2007). Londres Latina. La presencia colombiana en la capital británica (copia del autor).

Guzmán Ordaz, R. (2011). De la perspectiva de género al paradigma interseccional. Aportaciones para el análisis de las migraciones feminizadas. En Vázquez Bermúdez, I. (ed.). Logros y retos: Actas de III Congreso Universitario Nacional "Investigación y género". Sevilla: Universidad de Sevilla.

Gutiérrez De Pineda, V. (1997). La familia en Colombia. El trasfondo histórico. Medellín: Universidad de Antioquia.

Herrera, G. (2002). La migración vista desde el lugar de origen. Iconos, Revista de Ciencias Sociales, $15,86-94$

Hondagneu Sotelo, P. (1994). Gendered transitions: Mexican experiences of immigration. Berkeley: University of California Press.

Hondagneu Sotelo, P. y Avila, E. (1997). I'm here, but I'm there: The meaning of Latin transnational motherhood. Gender and Society, 11, 548-565.

Hochschild, A.R. (2001). Global care chains and emotional surplus value. En Hutton, W. y Giddens, A. (eds.). On the Edge. Living with Global Capitalism (pp. 130-146). Londres: Vintage.

Hurtado Saa, T. (2008). Movilidades, identidades y sexualidades en mujeres afrocolombianas migrantes en Europa: el caso de las italianas. En VV.AA., Raza, etnicidady sexualidades. Ciudadanía y multiculturalismo en América Latina. Bogotá: Universidad Nacional de Colombia, Facultad de Ciencias Humanas - Centro de Estudios Sociales - Centro de Estudios de Género.
ISTAT (2013). Stereotipi, rinunce e discriminazioni di genere. Anno 2011. Roma: ISTAT.

Jodelet, D. (1986). La representación social: fenómeno, concepto y teoría. En S. Moscovici. Psicología Social, 2, 469-495. Barcelona: Paidós.

Kóczé, A. (2011). La stérilisation forcé des femmes roms dans l'Europe d'aujourd'hui. Genre, modernité et "colonialité" du pouvoir. Cahiers du Gendre, 50, 133-152.

Kofman, E., Phizacklea, A., Raghuram, P. y Sales, R. (2000). Gender and international migration in Europe. London - New York: Routledge.

Lanzoni, S. (ed.) (2014). Rapporto sull'attuazione della Piattaforma d'Azione di Pechino. Rilevazione quinquennale: 2009-2014. Cosa veramente è stato fatto in Italia. Documento de Trabajo. Milán: Fondazione Pangea Onlus.

Lagomarsino, F. (2006). Esodi e approdi di genere. Famiglie transnazionali e nuove migrazioni dall'Ecuador. Milán: Franco Angeli.

Lutz, H., Herrera Vivar M.T. y Supik, L. (eds.) (2011). Framing intersectionality. Debates on a multifaceted concept in gender studies. Surrey: Ashgate.

Mahler, S.J. y Pessar, P. (2001). Gendered geographies of power: Analyzing gender across transnational spaces. Identities, 7(4), 441-459.

Merla, L. (2014). La circulación de cuidados en las familias transnacionales. Revista CIDOB d'Afers Internacionals, 106-107, 85-104.

Morokvasic, M. (1984). Bird of passage are also women. International Migration Review, 18(68), 886-907.

Morokvasic, M. (2008). Femmes et genre dans l'étude des migrations: un regard retrospectif. Les cahiers du Cedref, 16. Recuperado de http://cedref.revues.org/575

OIM (2012). Perfil migratorio de Colombia. Bogotá: OIM. 
Oso, L. (2000). L'immigration en Espagne des femmes chefs de famille. Les cahiers du CEDREF, 8-9. Recuperado de http://cedref.revues.org/191

Oso, L. y Catarino, C. (1997). Les effects de la migration sur le statut des femmes: le cas des Dominicaines et des Marocaines à Madrid et des cap-verdiennes à Lisbonne. Migration Societé, 9(52), 115-130.

Pachón, X. (2007). La familia en Colombia a lo largo del siglo XX. En Puyana, Y. y Ramírez, M. I. (eds.). Familias, cambios y estratégias (pp. 145-159). Bogotá: Universidad Nacional de Colombia, Colección CES.

Pardo, F. (2013). Inmigración, multiculturalidady politicas de integración en Europa. Colombianos en Amsterdam, Londres y Madrid. Bogotá: Universidad Externado de Colombia.

Parella, S. (2005). La maternidad a distancia de las empleadas domésticas latinoamericanas en España. La vulneración del derecho a la vida familiar en el contexto de la internacionalización de la reproducción. En Giró, J. (ed.). El género quebrantado. Sobre la violencia, la libertad y los derechos de la mujer en el nuevo milenio (pp. 283-273). Madrid: Catarata.

Parreńas Salazar, R. (2010). Transnational mothering: A source of gender conflicts in the family. North Carolina Law Review, 88(5), 1826-1855.

Parreñas Salazar, R. (2005). Long distance intimacy: class, gender and intergenerational relations between mothers and children in Filipino transnational families. Global Networks, 5(4), 317-336.

Parreñas Salazar, R. (2001). Servants of Globalization: Woman Migration and Domestic Work. Stanford: Stanford University Press.

Pedone, C. (2008). "Varones aventureros" vs. "Madres que abandonan": Reconstrucción de las relaciones familiares a partir de la migración ecuatoriana”.
REMHU Revista Insterdisciplinar da Mobilidade Humana, XVI (30), 45-64.

Pedone, C. (2006). Estrategias migratorias y poder. Tú siempre jalas a los tuyos. Quito: Ediciones Abya Yala.

Pedone, C, Echeverri, M. M. y Gil Araújo, S. (2014). Entre dos orillas. Cambios en las formas de organización de las familias migrantes latinoamericanas en España en tiempos de crisis global. En Zabala de Cosío, M. E. y Rozée Gomez, V. (eds.). El género en movimiento. Familias y migraciones (109-138). México: El colegio de México, Centro de Estudios Demográficos, Urbanos y Ambientales.

Piccone, S. y Saraceno, C. (1996). Genere. La costruzione sociale del femminile e del maschile. Bolonia: Il Mulino.

Phizacklea, A. (ed.) (1983). On Way Ticket. Migration and Female Labour. Londres: Routledge y Kegan Paul.

Posso Quiceno, J. (2007). La migración colombiana hacia España, las redes de hogares trasnacionales y los cambios en las relaciones de género. Amérique Latine Histoire et Mémoire. Les Cahiers ALHIM, 14. Recuperado de http://alhim.revues.org/index2162.html - ftn 1

Profamilia (2010). Encuesta Nacional de Demografía y Salud - ENDS 2010. Recuperado de http:// www.profamilia.org.co/encuestas/Profamilia/ Profamilia/

Puyana Villamizar, Y. (ed.) (2003). Padres y madres en cinco ciudades colombianas. Cambios y permanencias. Bogotá: Almuneda Editores.

Puyana Villamizar, Y., Micolta León, A. y Palacio, M. C. (eds.) (2013). Familias colombianas y migración internacional: entre la distancia y la proximidad. Bogotá: Universidad Nacional de Colombia, Colección CES.

Rivas Rivas, A. M. y Gonzálvez Torralbo, H. (eds.) (2009). Familias transnacionales colombianas. 
Transformaciones y permanencias en las relaciones familiares y de género. Madrid: Catarata.

Saraceno, C. (2005). Paternità e maternità. Non solo disuguaglianze di genere. Comunicación presentada en el Seminario "La paternità in Italia". Roma.

Saraceno, C. (1996). Sociologia della famiglia. Bolonia: Il Mulino.

Scott, J. (1986). El género: Una categoría útil para el análisis histórico. En Lamas, M. (ed.). El género: la construcción cultural de la diferencia sexual (pp. 265-302). México: PUEG.

Sinatti, G. (2014), Masculinities and intersectionality in migration: Transnational Wolof migrants negotiating manhood and gendered family roles. En Truong, T. D. et al. (eds.). Migration, gender and social justice: Perspectives on human insecurity. Hexagon Series on Human and Environmental Security and Peace, 9, 215-226.

Parella, S. y Cavalcanti, L. (2007). Los vínculos económicos y familiares transnacionales. Los inmigrantes ecuatorianos y peruanos en España. En Solé, C. (ed.). Informes de Ciencias Sociales. Bilbao: Fundación BBVA.

Tienda, M. y Booth, K. (1991). Gender, migration and Social Change. International Sociology, 6(1), 51-72.

Wagner, H. (2008). Maternidad transnacional: discursos, estereotipos, prácticas. En Herrera, G. y Ramírez, J. (eds.). América Latina migrante: Estado, familia, identidades. Quito: FLACSO.

Yuval Davis, N. (2006). Intersectionality and feminist politics. European Journal of Women' Studies, 13(3), 193-209.
World economic forum (2013). Global Gender Gap Report 2013. Recuperado de http://www.weforum. org/issues/global-gender-gap

Zlotnik, H. (1992). Empirical identification of international migration systems. En Kritz, L. y Zlotnik, H. (eds.). International Migration Systems. Oxford: Clarendon Press.

Zlotnik, H. (2003). The global dimension of female migration. Migration Information Source. Recuperado de http://www.migrationpolicy.org/article/ global-dimensions-female-migration

Zontini, E. (2005). Migraciones, género y multiculturalismo. Una perspectiva de Europa meridional. En Nash, M., Tello, R. y Benach, N. (eds.). Inmigración, género y espacios urbanos. Los retos de la diversidad. Barcelona: Edicions Bellaterra.

\section{Sitios web}

ISTAT - Istituto Nazionale di Statistica

Immigrati.Stat: dati e indicatori su immigrati e nuovi cittadini, http://stra-dati.istat.it/

Demografia in cifre, http://demo.istat.it/

DANE - Departamento Administrativo de Estadística, http://www.dane.gov.co/

Colombia Nos Une - Cancillería Ministerio de Relaciones Exteriores http://www.cancilleria.gov.co/ footer/join-us, http://www.redescolombia.org/ oIM Colombia, http://www.oim.org.co/

Ministero dell'Interno - Immigrazione e Asilo, http:// www.interno.gov.it/it/temi/immigrazione-e-asilo 